\title{
Emerging Roles of N6-Methyladenosine Demethylases and Its Interaction with Environmental Toxicants in Digestive System Cancers
}

\author{
Caiping Liu' \\ Sheng Yang' \\ Yanqiu Zhang ${ }^{2}$ \\ Chuntao Wang ${ }^{3}$ \\ Dandan Du' \\ Xian Wang' \\ Tong Liu (D)' \\ Geyu Liang'
}

'School of Public Health, Key Laboratory of Environmental Medicine Engineering,

Ministry of Education, Southeast

University, Nanjing, 210009, Jiangsu,

People's Republic of China; ${ }^{2}$ Department

of Environmental Occupational Health,

Taizhou Center for Disease Control and

Prevention, Taizhou, 225300, Jiangsu,

People's Republic of China; ${ }^{3}$ jiangsu

Vocational College of Medicine,

Yancheng, 224000, Jiangsu, People's

Republic of China
Correspondence: Geyu Liang

Tel +862583272572

$\mathrm{Fax}+862583324322$

Email lianggeyu@163.com

\begin{abstract}
Digestive system cancers are common cancers with high cancer deaths worldwide. They have become a major threat to public health and economic burden. As one of the most universal RNA modifications in eukaryotes, the N6-methyladenosine (m6A) modification is involved in the occurrence, development, prognosis, and treatment response of various cancers, including digestive system cancers. M6A demethylases shape the m6A landscape dynamically, playing important roles in cancers. In addition, accumulating evidence reveal that many environmental toxicants are the established risk factors for digestive system cancers and associated with m6A modification. In this review, we summarize the multiple functions of M6A demethylases (fat mass and obesity-associated protein (FTO), AlkB homolog 5 (ALKBH5) and AlkB homolog 3 (ALKBH3)) in digestive system cancers, which are aberrantly expressed and affect cancer progression. We also discuss the potential roles of m6A demethylases in the assessment of environmental exposure, the signature for prevention and diagnosis of digestive system cancers.
\end{abstract}

Keywords: m6A modification, FTO, ALKBH5, environment toxicants, digestive system cancers

\section{Introduction}

With the rapid growth and aging of the population, cancer has occupied the primary reasons for death that placed a huge personal and societal healthcare burden. ${ }^{1,2}$ Global Cancer Statistics showed 19.3 million new cancer cases and 10.0 million cancer deaths in 2020, and the number of global new cancer cases is expected to grow to 28.4 million by $2040 .^{3}$ At present, it has been generally agreed that the interaction of genetic and environmental factors promote the occurrence and development of cancers, but the accurate mechanism is still unclear. The digestive system cancers are common malignant cancers with a poor prognosis and high mortality, because most digestive system cancers are usually discovered and diagnosed at their advanced stage which leads to the treatment effect not being obvious, ${ }^{4}$ and patients with higher risk to go suicidal when suffering from cancer in psychological aspects. ${ }^{5}$ The five most common digestive system cancers, including stomach cancer, liver cancer, esophagus cancer, pancreatic cancer and colorectal cancer, account for $30.7 \%$ and $35.4 \%$ of all cancer incidence and mortality, ${ }^{3}$ respectively. Therefore, digestive system cancers have become one of the most concerned public health problems. 
Massive progress has been made on the research of the cancer molecular mechanism which gained much attention in the last decade. RNA modifications are one of the heaviest mechanisms of cancers, and more than 170 RNA modifications have been identified. ${ }^{6} \mathrm{M} 6 \mathrm{~A}$ is the methylation modification of the sixth nitrogen $(\mathrm{N})$ atom of adenine (A) occupied for more than $60 \%$ of RNA modifications, which is the most abundant modification of RNA in eukaryotes. ${ }^{7}$ It was first identified in the 1970s, but m6A did not get any further over the following years. ${ }^{8}$ Until the recent decade, with the high-throughput detecting techniques developing and the oxidative demethylases of m6A uncovering, m6A was made clear as a dynamic and reversible process and became a cancer research hotspot. Increasing studies show that $\mathrm{m} 6 \mathrm{~A}$ modifications prefer to occur in the consensus motif of RRACH $(\mathrm{R}=\mathrm{G}$ or $\mathrm{A}$; $\mathrm{H}=$ $\mathrm{A}, \mathrm{C}$, or $\mathrm{U}$ ), which mainly enrich in stop codons, $3^{\prime}$ untranslated region (3' UTR) and the exon in RNA. ${ }^{9}$ With the advance of m6A, the studies demonstrate that m6A and $\mathrm{m} 6 \mathrm{~A}$ associated proteins are involved in various biological and pathological processes, such as splicing, transport, translation and degradation. ${ }^{10,11}$ In addition, the abnormal expression of $\mathrm{m} 6 \mathrm{~A}$ associated proteins are involved in regulating genes that impact cell processes and physiological function in various cancers. As Figure 1 shows, m6A modification is regulated by three types of proteins, including the m6A methyltransferase ("writer"), demethylase ("eraser") and reading proteins ("reader"). The m6A methyltransferase ("writer"), METTL3, METTL14, WTAP and other proteins formed a multicomponent methyltransferase complex (MTC), that catalyzes the formation of methylation on RNA. And the RNA binding protein ("reader") YTH domain family (YTHDF1-3, YTHDC1 and YTHDC2), Insulin-like growth factor 2 mRNA-binding proteins 1-3 (IGF2BP1-3), Heterogeneous nuclear ribonucleoprotein (HNRNPC, HNRNPA2B1) and other proteins can recognize methylation on RNA. ${ }^{12}$ The m6A demethylases ("eraser"), such as FTO, ALKBH5 and ALKBH3, share a common mechanism to remove the m6A modification. ${ }^{13}$ The expression of m6A demethylases in mRNA and protein levels are often significantly different between tumor tissues and the adjacent normal tissues, ${ }^{14}$ and influence greatly on cell function ${ }^{15,16}$ by regulating downstream target genes, ${ }^{17}$ which also plays important roles in progression and treatment of various cancers, such as respiratory system cancers, ${ }^{18}$ reproductive system cancers, ${ }^{19}$ urinary system cancers ${ }^{20}$ and digestive system cancers. $^{21}$ It is known that $\mathrm{N}$-nitroso compounds, Helicobacter pylori (HP) infection, tobacco smoking, excess alcohol, aflatoxin and aristolochic acid are established risk factors for digestive system cancers. ${ }^{22,23}$ When exposed to the environmental toxicant, m6A methylation levels and m6A demethylase expression can alter with a time- and dose-dependent manner, ${ }^{24}$ playing critical roles in cancers induced by various environmental toxicants. Given the evidence that has accumulated, it is

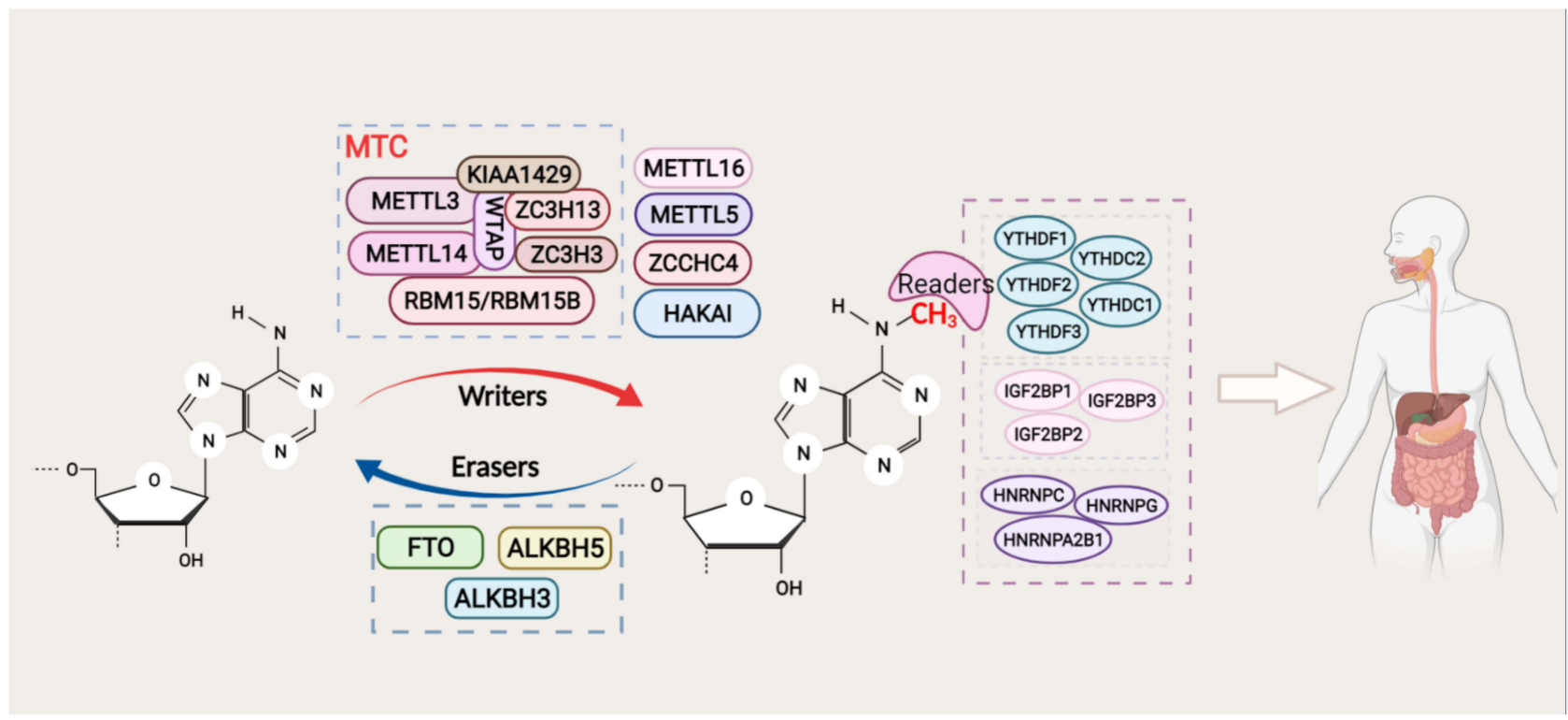

Figure I The dynamics and reversible process of m6A modification. M6A is reversed by m6A demethylases (FTO, ALKBH5 and ALKBH3) and plays important roles in digestive system cancers. 
categorically stated that the m6A demethylases can regulate RNA m6A methylation levels, thereby affecting the occurrence and development of cancers by complex signal pathways, indicating that m6A demethylases can serve as potential biomarkers for diagnosis, therapy and prognosis of digestive system cancers.

In this review, we first provided comprehensive insights into the roles and molecular mechanism of m6A demethylases in digestive system cancers. More importantly, we also understood the association between m6A methylation levels and environmental toxicants in digestive system cancers and highlighted their potential clinical applications in future cancer diagnosis and treatment.

\section{Role of the FTO Gene in Digestive System Cancers The Discovery of FTO}

Fat mass and obesity-associated protein (FTO) also known as alkB homolog 9 (ALKBH9), is lied at the 16 chromosomes and belongs to the a-ketoglutaric acid $(\alpha-K G)$ dependent ALKB family of dioxygenases. FTO is highly expressed in the brain, adrenal and thyroid tissues. It was initially discovered in mice and contributed to human obesity and energy by Genome-Wide Association Studies (GWAS) analysis. ${ }^{25}$ FTO is strongly associated with obesity that can result in multiple diseases, including heart disease, ${ }^{26}$ type 2 diabetes and cancers. ${ }^{27}$ Some studies found that FTO-related single nucleotide polymorphism (SNP) was involved in breast cancer, ${ }^{28}$ endometrial cancer and pancreatic cancer. ${ }^{29,30}$ In 2011, FTO was demonstrated to be as a demethylase of m6A modification and the dynamic reversible process was proved. ${ }^{31}$ Wei et al ${ }^{32}$ justified that FTO was not only restricted in the nucleus but also in the cytoplasm, and the location of FTO was closely correlated to its function. In 2017, the evidence suggested that FTO played an oncogenic role in Acute Myeloid Leukemia (AML) as an m6A RNA demethylase. ${ }^{33}$ Since then, it has opened the research of FTO as an m6A demethylase in cancers, which provided new clues for the research of cancers molecular mechanism.

\section{FTO Dysregulation in Digestive System Cancers Gastric Cancer (GC)}

$\mathrm{GC}$ is one of the most common malignant cancers with high incidence, high mortality and poor prognosis, whose incidence and mortality rate ranked third and second of all cancers in China in 2018, respectively. ${ }^{4}$ Several studies proved that the expression of FTO was up-regulated and correlated with the m6A level of mRNA in GC by the The Cancer Genome Atlas (TCGA) and Gene Expression Omnibus (GEO) database analysis. ${ }^{34,35}$ In addition, FTO was also over-expressed in GC samples from Chinese cohort by performing RT-qPCR, Western blot and immunohistochemistry (IHC), showing good agreement with the results of databases. ${ }^{36,37}$ And the epidemiological and clinical characteristics investigations revealed that the FTO expression was associated with age, differentiation, lymph node metastasis, TNM stage, and prognosis in GC patients. $^{14,38,39}$ Beyond that, increased expression of FTO can reduce $\mathrm{m} 6 \mathrm{~A}$ methylation and regulate $\mathrm{MYC}$ to promote the cell proliferation, migration and invasion ability in GC by FTO/m6A/MYC molecular network. ${ }^{40,41}$

However, several results of studies were inconsistent with the above results. They affirmed that FTO was lower expressed in GC and served as an anti-oncogene to involve in cell proliferation, invasion and migration. ${ }^{42,43}$ Moreover, it is reported that the level of m6A in peripheral blood of patients with GC was increased, accompanied by the downregulation of FTO, which could provide promising noninvasive biomarkers for $\mathrm{GC}$ diagnosis. ${ }^{44}$ It was worth noting that one study revealed that the expression of FTO was different between mRNA and protein levels. FTO was over-expressed at mRNA level but it was markedly downregulated at the protein level in GC tissue. ${ }^{42}$ Above all, the FTO expression is different in various cohorts because of the different source of samples and the various post-transcriptional regulation mechanisms, which should be further elucidated in large cohorts.

\section{Liver Cancer}

Hepatocellular carcinoma (HCC) and intrahepatic cholangiocarcinoma (ICC) are primary liver cancers with high mortality. As an anti-oncogene gene, FTO played a protective function in liver cancers. ${ }^{45,46}$ Rong et $\mathrm{al}^{45}$ found that the expression of FTO was downregulated in ICC samples and cell lines to influence tumor growth by impairing oncogene TEAD2 mRNA stability. Moreover, low level of FTO expression could predict poor prognosis in ICC. ${ }^{45}$ Liu et al $^{46}$ also demonstrated that FTO was decreased in HCC samples and interacted with SIRT1 to influence HCC progression. Several studies hold conflicting opinions against above results, suggesting that FTO was overexpressed in HCC and could promote proliferation and invasion. ${ }^{4,48}$ According to a long-term mice 
experiment, FTO is essential for the control of energy balance and glucose metabolism. Importantly, during the initiation phase of tumor development, FTO is upregulated on acute liver damage but plays protective roles in HCC development. ${ }^{49}$ Therefore, FTO can be dynamically regulated in different periods of the liver cancer development, which can serve as a diagnostic marker and therapeutic target in liver cancer.

\section{Colorectal Cancer (CRC)}

The global cancer statistic of 2020 showed that the incidence and mortality rate of colorectal cancer (including colon cancer and rectal cancer) ranked third and second, ${ }^{3}$ respectively. FTO is upregulated in CRC and reduces the m6A modification, which can active MYC to induce carcinogenesis and regulate PD-L1 to affect immune escape. ${ }^{50,51}$ Besides, low expression of FTO was related to polyubiquitin binding, mRNA 3' end processing, transcription elongation from RNA polymerase II and poor overall survival. ${ }^{52,53}$ In addition, diminished FTO expression is the key factor for promoting the cancer stem-like traits in $\mathrm{CRC}$, including sphere forming, in vivo tumorigenicity, and chemoresistance. Compared to primary and metastatic tumor cells, FTO expression is also lower in circulating tumor cells, which can serve as diagnostic biomarkers for CRC. ${ }^{54}$ Therefore, FTO is important for improving diagnosis, maintaining cancer stem cell phenotype, assessing treatment effect and predicting prognosis in CRC.

\section{Other Digestive System Cancers}

In addition to the above common digestive system cancers, FTO is also involved in the development of pancreatic cancer (PC) and esophageal cancer (EC). A high level of FTO expression was confirmed in $\mathrm{PC}$, which is necessary for tumor growth by regulating the downstream target c-MYC. ${ }^{55}$ FTO is also highly expressed in EC, which can promote cell proliferation and migration by up-regulating MMP13. ${ }^{56,57}$

The expression of FTO is complex in digestive system cancers, playing an essential role in tumor occurrence and development. In addition, MYC may be an important downstream target of FTO, whose expression and stability are regulated by FTO-mediated m6A modification. Generally, FTO and its target genes provide new insight into diagnosis and treatment for digestive system cancers (as can be seen in Table 1).

\section{Role of the ALKBH5 Gene in Digestive System Cancers}

\section{The Discovery of ALKBH5}

ALKBH5 is also known as ABH5 or OFOXD. It is located on the 17 Chromosome. ALKBH5 is a member

Table I The Role of FTO in Human Digestive System Cancers

\begin{tabular}{|c|c|c|c|c|c|}
\hline Cancer Types & Roles & Expression & Target & Molecular Mechanism & References \\
\hline Gastric cancer & Oncogene & $U_{p}$ & MYC & $\begin{array}{c}\mathrm{HDAC} 3 \text { mediated } \mathrm{FTO} / \mathrm{m} 6 \mathrm{~A} / \mathrm{myc} \text { axis to regulate } \mathrm{FOXA} 2 \text { in GC } \\
\text { initiating activities. }\end{array}$ & [40] \\
\hline $\begin{array}{l}\text { Intrahepatic } \\
\text { cholangiocarcinoma }\end{array}$ & Suppressor & Down & GNAOI & $\begin{array}{l}\text { Depleted FTO by SIRTI promoted m6A+ levels of HCC tumor } \\
\text { suppressor GNAOI and decreased its mRNA expression. }\end{array}$ & [46] \\
\hline $\begin{array}{l}\text { Hepatocellular } \\
\text { carcinoma }\end{array}$ & Oncogene & $U_{p}$ & PKM2 & $\begin{array}{l}\text { FTO triggered the demethylation of PKM2 mRNA and accelerated } \\
\text { the translated production. }\end{array}$ & [47] \\
\hline $\begin{array}{l}\text { Hepatocellular } \\
\text { carcinoma }\end{array}$ & Suppressor & - & Cul4a & $\begin{array}{l}\text { FTO-dependent m6A regulated of Cul4a mRNA to play protective } \\
\text { function in the initiation of } \mathrm{HCC} \text { development. }\end{array}$ & [49] \\
\hline Colorectal cancer & Oncogene & $U_{p}$ & PD-LI & $\begin{array}{l}\text { FTO mediated } \mathrm{m} 6 \mathrm{~A} \text { regulated PD-LI expression that might affect } \\
\text { a therapeutic response to immunotherapy. }\end{array}$ & [50] \\
\hline Colorectal cancer & Oncogene & Up & MYC & $\begin{array}{l}\text { MiR-96 could potentially stimulate malignancy and aggressiveness } \\
\text { of CRC by activating AMPK } \alpha 2 \text {-mediated FTO/MYC. }\end{array}$ & [5। $]$ \\
\hline Pancreatic cancer & Oncogene & $U_{p}$ & c-MYC & $\begin{array}{l}\text { FTO enhanced stability of MYC and bHLH via decreasing m6A } \\
\text { level. }\end{array}$ & [55] \\
\hline $\begin{array}{l}\text { Esophageal } \\
\text { squamous cell } \\
\text { carcinoma }\end{array}$ & Oncogene & Up & MMPI3 & $\begin{array}{l}\text { FTO facilitated cell proliferation and migration by up-regulating } \\
\qquad \text { MMPI3. }\end{array}$ & [57] \\
\hline
\end{tabular}


of the $\alpha-\mathrm{KG}$ dependent ALKB family of dioxygenases, it was identified as the second demethylases in 2013 . $^{58}$ FTO is not only located in the nucleus, but also in the cytoplasm in some cases, but most of ALKBH5 is located in the nuclear speckles with different roles according to the site of action. ${ }^{32}$ Both FTO and ALKBH5 rely on $\mathrm{Fe}$ (II) and $\alpha$ ketoglutaric acid in different ways during the demethylation of m6A. The demethylation of m6A catalyzed by FTO can be divided into two steps. First, the demethylation of m6A to N6hydroxymethyladenosine (hm6A) is catalyzed by FTO, and then hm6A is converted to N6-formyladenosine (f6A) and further oxidized to product A. ${ }^{12}$ But ALKBH5 can directly catalyze m6A to A with no intermediate products.

\section{The Role of ALKBH5 Dysregulation in Digestive System Cancers Gastric Cancer}

To evaluate the function of AlkB homolog 5 (ALKBH5), the researchers have drawn conclusions based on the TCGA database that ALKBH5 was an independent indicator to predict prognosis of GC patients. ${ }^{34,38,39,59}$ In addition, ALKBH5 is overexpressed in GC and demethylates NEAT1 to promote invasion and migration by regulating EZH2 ${ }^{60}$ However, the level of m6A in peripheral blood of GC patients is increased with down-regulated ALKBH5, which also is associated with progression and metastasis. ${ }^{44}$

\section{Colorectal Cancer}

Currently, several studies have reported the relationship between ALKBH5 and colorectal cancer. According to the different CRC cohorts, ALKBH5 is low expression in CRC patients at mRNA and protein levels, playing a suppressor gene role in CRC. ${ }^{61-64}$ Besides, the expression of ALKBH5 is significantly associated with age, stage, invasion and metastasis, overall survival and disease-free survival, which is also verified by experiments in vitro and in vivo. ${ }^{64}$ However, some scholars argue that ALKBH5 is upregulated in CRC tissues and cells. ${ }^{53,65}$ Guo et al emphasized that ALKBH5 expression was increased in CRC cells, and the cell proliferation, migration were impeded and apoptosis was improved by ALKBH5-NEAT1 axis, which might be a potential therapeutic target for colon cancer treatment. ${ }^{65}$ At the moment, there are still some controversy about the role of ALKBH5, requiring further discussion.

\section{Pancreatic Cancer}

As the results of increasing morbidity and mortality of PC with $7 \%$ five-year survival rate, ${ }^{66}$ there is an urgent need to find the biomarker for diagnosis, intervention and treatment. According to the multi-cohort analysis, we can conclude that the ALKBH5 is downregulated in PC and has better discriminatory power than other clinical variables, which can also predict overall survival. ${ }^{67,68}$ Increasing evidence substantiated that down-regulated ALKBH5 can promote cell proliferation, migration, invasion and tumor growth ability, and vice versa. ${ }^{69-71}$ Several mechanisms were investigated to explain it. Tang et al confirmed that ALKBH5 could inhibit the activation of Wnt signaling pathway by reducing the level of m6A of downstream target WIF-1, and ultimately repressed tumor development in vivo and in vitro. ${ }^{69}$ Similar to regulating WIF-1, ALKBH5 can activate ATM-CHK2P53/CDC25C signal pathway by regulating PER1 and form ALKBH5-PER1-P53-ALKBH5 feedback loop to influence m6A methylation. ${ }^{70}$ In addition, ALKBH5 also can interact with non-coding RNA dependent on demethylating to involve in the occurrence and progress of pancreatic cancer. ${ }^{71}$ Many studies reported that ALKBH5 was related to the infiltration of immune cells, which was helpful to determine the targets of immunotherapy aimed at inhibiting tumorigenesis. $^{72,73}$ In summary, ALKBH5 may be a potential target for diagnosis and therapy of $\mathrm{PC}$ in the future.

\section{Other Digestive System Cancers}

As for liver cancer, the mutation and copy number variation of ALKBH5 have clear relation with clinicopathological features and prognosis of patients. ${ }^{74}$ Chen et al found that ALKBH5 was down-regulation and attenuated the expression of LYPD1 via an m6A-dependent manner to promote the cell biological effects in HCC. ${ }^{75}$ To better explain this mechanism, the author knocked-down the FTO expression and found it had almost no influence on LYPD1, suggesting that ALKBH5 and FTO as powerful m6A demethylases both could effectively demethylate m6A, but the ALKBH5 demethylation capacity was different from FTO. ${ }^{75}$ On the one hand, ALKBH5 is as a suppression gene and decreases in the EC. ${ }^{76,77}$ ALKBH5 can form a positive feedback loop with miR$193 a-3 p$ to prevent pri-miR-193a-3p maturation and regulate tumor growth in vivo and in vitro. ${ }^{77}$ But on the other hand, ALKBH5 plays an oncogene role and mediated m6A modification to increase CDKN1A mRNA stability in $\mathrm{EC}^{78}$ 
The aforementioned studies indicate that the function and regulatory mechanisms of ALKBH5 are diverged in most digestive system cancers. Similar to FTO, ALKBH5 serves as the demethylase of m6A demethylase and plays different functions and regulatory mechanisms in various digestive system cancers (as shown in Table 2), which is still unclear and needs further study.

\section{The Role of ALKBH3 Dysregulation in Digestive System Cancers}

AlkB homolog 3 (ALKBH3) is also known as ABH3, PCA1 and DEPC-1. It is located on the human 11 chromosomes, existing in both cell cytoplasm and nucleus. ALKBH3 was first identified as 1-methyladenosine $\left(\mathrm{m}^{1} \mathrm{~A}\right)$ and 3-methylcytidine $\left(\mathrm{m}^{3} \mathrm{C}\right)$ demethylase of RNA in human ALKB homolog. ${ }^{79}$ And several studies confirmed that $\mathrm{ALKBH} 3$ as $\mathrm{m}^{1} \mathrm{~A}$ and $\mathrm{m}^{3} \mathrm{C}$ demethylase of tRNA which promoted the cancer cell proliferation, migration and invasion and affected tumor growth. ${ }^{6,80}$ Ueda et al recently verified that ALKBH3 was m6A demethylase of
tRNA that improved the efficiency of protein translation and related to tumor growth and proliferation. ${ }^{81}$ ALKBH3, as one of the m6A demethylases, has come to light only recently, and less study is focused on it. ALKBH3 may be direction and priorities of research in the future.

M6A is reversed by m6A demethylases (FTO, ALKBH5 and ALKBH3) and plays important roles in digestive system cancers. The expression levels of m6A demethylases vary in different normal tissues or turn malignant tissues. And m6A demethylases perform their biological function to involve in tumor occurrence and development through m6A dependent mechanisms in digestive system cancers, as can be seen in Figure 2.

\section{The Role of Demethylase in Digestive System Cancers Induced by the Environmental Toxicants}

Environmental toxicants are widespread in natural environment and living conditions. Substantial epidemiological investigations and studies confirmed that the

Table 2 The Role of ALKBH5 in Human Digestive System Cancers

\begin{tabular}{|c|c|c|c|c|c|}
\hline Cancer Types & Roles & Expression & Target & Molecular Mechanism & References \\
\hline Gastric cancer & Oncogene & Up & NEATI & $\begin{array}{l}\text { ALKBH5 promoted GC invasion and metastasis by demethylating } \\
\text { the IncRNA NEATI. }\end{array}$ & [60] \\
\hline Colon cancer & Suppression & Down & - & $\begin{array}{l}\text { Overexpression of ALKBH5 inhibited colon cancer cells invasion } \\
\text { in vitro and metastasis in vivo. }\end{array}$ & [64] \\
\hline Colon cancer & Oncogene & Up & NEATI & ALKBH5-NEATI axis might regulate malignant behavior. & [65] \\
\hline $\begin{array}{l}\text { Pancreatic } \\
\text { cancer }\end{array}$ & Suppression & Down & WIF-I & $\begin{array}{l}\text { ALKBH5 repressed PDAC tumorigenesis by reducing m6A levels of } \\
\text { WIF-I and hindering activation of Wnt signaling. }\end{array}$ & [69] \\
\hline $\begin{array}{l}\text { Pancreatic } \\
\text { cancer }\end{array}$ & Suppression & Down & PERI & $\begin{array}{l}\text { ALKBH5 activated PERI by m6A demethylation in an m6A- } \\
\text { YTHDF2-dependent manner and P53-induced activation of } \\
\text { ALKBH5 transcription acted as a feedback loop regulating the } \mathrm{m} 6 \mathrm{~A} \\
\text { modifications in PC. }\end{array}$ & [70] \\
\hline $\begin{array}{l}\text { Pancreatic } \\
\text { cancer }\end{array}$ & Suppression & Down & $\begin{array}{l}\text { KCNKI5- } \\
\text { ASI }\end{array}$ & $\begin{array}{l}\text { ALKBH5 mediated m6A to regulate KCNKI5-ASI and affect its } \\
\text { stability. }\end{array}$ & [7I] \\
\hline $\begin{array}{l}\text { Hepatocellular } \\
\text { carcinoma }\end{array}$ & Suppressor & Down & LYPDI & $\begin{array}{l}\text { Down-regulation of ALKBH5 activated the m6A machinery } \\
\text { contributing to the epigenetic activation of LYPDI which is } \\
\text { recognized and stabilized by IGF2BPI. }\end{array}$ & [75] \\
\hline $\begin{array}{l}\text { Esophageal } \\
\text { squamous cell } \\
\text { carcinoma }\end{array}$ & Suppressor & Down & $\begin{array}{l}\text { Pri-miR } \\
-193 a-3 p\end{array}$ & $\begin{array}{l}\text { ALKBH5 mediated m6A modification to regulate the pri-miR-193- } \\
\text { 3p processing which suggested a positive feedback loop between } \\
\text { miR-193-3p and ALKBH5. }\end{array}$ & [77] \\
\hline $\begin{array}{l}\text { Esophageal } \\
\text { squamous cell } \\
\text { carcinoma }\end{array}$ & Oncogene & Up & CDKNIA & $\begin{array}{l}\text { Depletion of ALKBH5 increased m6A methylation and stability of } \\
\text { CDKNIA mRNA, leading to up-regulation of p2I (CDKNIA) } \\
\text { protein expression. }\end{array}$ & [78] \\
\hline
\end{tabular}




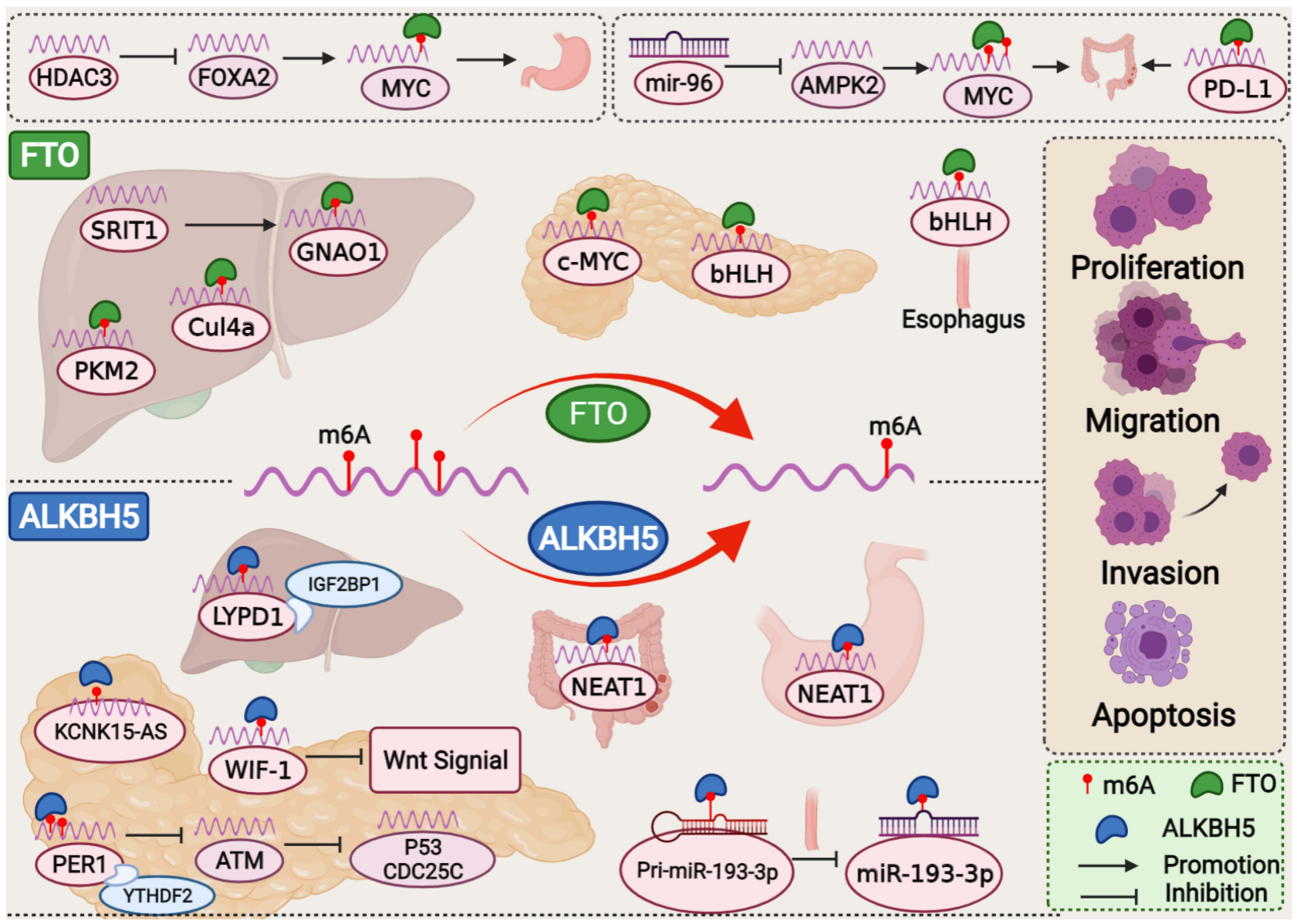

Figure 2 The mechanism and functions of m6A demethylases FTO and ALKBH5 involved in human digestive system cancers.

environmental factors induce multiple genetic and epigenetic changes that promote tumorigenesis. For example, N-nitroso compounds, $H P$ and others are risk factors for GC. ${ }^{22}$ Several risk factors have been identified for liver cancer, such as aflatoxin and aristolochic acid. ${ }^{23} H P$, hot food or drinks are risk factors for esophageal cancer. ${ }^{82}$ In addition, tobacco smoking and excess alcohol are common environmental risk factors for digestive system cancers. ${ }^{23,83}$ Identifying signature of the cancer induced by environmental risk factors and recognizing the highrisk population exposure to environmental toxicants can make it recognizable and preventable and reduce the risk of digestive system cancers. Currently, because of the lack of studies about environmental exposures and m6A mechanisms, only a minority of researches reveal the level of m6A methylation are in response to environmental toxicants exposure, as shown in Figure 3. Han et al affirmed that the levels of $\mathrm{m} 6 \mathrm{~A}$ and $\mathrm{m} 6 \mathrm{~A}$ related proteins were significantly decreased and activated the PI3K/Akt/ mTOR pathway to promote the cell proliferation and aggravate pulmonary fibrosis in carbon black (CB)treated rats. ${ }^{84}$ The level of $\mathrm{m} 6 \mathrm{~A}$ is down-expressed and significantly affects the role and expression of non-coding RNAs in the ovarian injury induced by Cadmium $(\mathrm{Cd}) .{ }^{85}$ In addition, the study found that the levels of global m6A in peripheral blood was downregulated in smokers compared with non-smokers, but they were up-regulated after acute $\mathrm{CB}$ exposure. ${ }^{86}$ On the contrary, the level of m6A was upregulated in arsenite-transformed lung cells by its methyltransferases and demethylase to affect miRNAs to involve in the arsenite-induced proliferation and apoptosis. ${ }^{87}$ Interestingly, Chen et al confirmed that the reversible $\mathrm{m} 6 \mathrm{~A}$ and $\mathrm{m} 6 \mathrm{~A}$ associated protein showed hormesis effect in the $\mathrm{NaAsO}_{2}$-induced keratinocyte cells. ${ }^{88}$ The m6A methylation alter various due to the diversity and complexity of environmental exposures.

The above studies confirmed the association between m6A methylation and environmental toxicant exposure, next we focus attention on m6A demethylases. The m6A levels are decreased with increased FTO and ALKBH5 in 


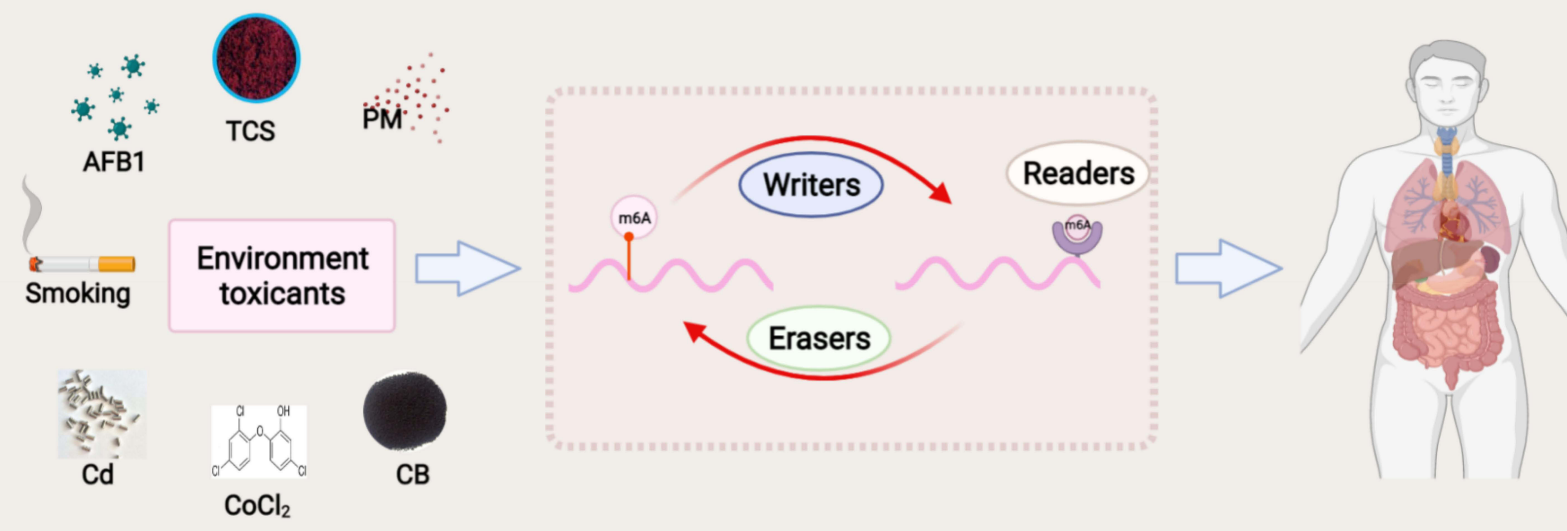

Figure 3 Effect of m6A modification on human diseases by environmental toxicants exposures.

lung adenocarcinoma cell after exposed to high-dose Particulate matter $\left(\mathrm{PM}_{2.5}\right){ }^{89} \mathrm{CoCl}_{2}$ exposure raises the expression of m6A demethylase and decreasing the activity of FTO and ALKBH5 in neurodegenerative diseases. ${ }^{90}$ After rats treated with Di-(2-Ethylhexyl) phthalate (DEHP), Nrf2-mediated antioxidant signaling pathway was inhibited in prepubertal testes, which increased m6A levels with FTO decreased. ${ }^{91}$ Cui et $\mathrm{al}^{92}$ found FTO and autophagy dysfunction form a positive feedback loop in chronic low-level arsenic exposure which impaired the expression of FTO and m6A. Moreover, m6A demethylases are also demonstrated to play important roles in digestive system diseases and cancers induced by environmental factors. Cigarette smoke condensate (CSC) exposure can induce miR-25-3p excessive maturation via m6A modification to promote the development and progression of PC. ${ }^{93}$ Aflatoxin B1 (AFB1) exposure can induce reactive oxygen species (ROS) accumulation to increase m6A expression, but which can be reversed by resveratrol treatment, paving an avenue for liver diseases prevention and treatment. ${ }^{94}$ It is known that some liver-associated metabolic diseases are caused by triclosan (TCS) and bisphenol A exposure (BPA), where the expression of FTO was ascended to increase lipogenesis and lipid transport and inhibit lipid oxidation with different regulation modes. ${ }^{95}$ Besides, FTO inactivation can increase diethylnitrosamine (DEN)-induced HCC burden, ${ }^{49}$ indicating protective function of FTO in liver carcinogenesis. Cigarette smoke exposure can increase ALKBH5 expression and mediate m6A to reduce the translation of LINC00278-sORF1 in EC progression. ${ }^{96}$

Given the potential significance alters of m6A and m6A associated protein in environmental factors-driven carcinogenesis, it provides new insights into the assessment of environmental exposure, the signature for early damage of digestive systems, and the prevention and diagnosis of digestive system cancers (as shown in the Table 3).

\section{Conclusion and Outlook}

Until now, a great number of studies have investigated the relationship between m6A demethylases and various cancers. It is confirmed that dysregulated m6A demethylases in the transcripts of some oncogenes or suppressors are involved in tumor progression and metastasis. Alternatively, because of the chemo-radiotherapy drug resistance characteristic, the researchers hoped to find inhibitor or preventive biomarkers to block tumor development of tumor based on m6A demethylase. Fortunately, some remarkable advances have been made on FTO and ALKBH5 inhibitors, ${ }^{97,98}$ which can become a key technique in the domain of cancer therapy. Therefore, it is important to develop highly specific and effective inhibitors of m6A demethylation for cancer therapeutic applications in the future. 


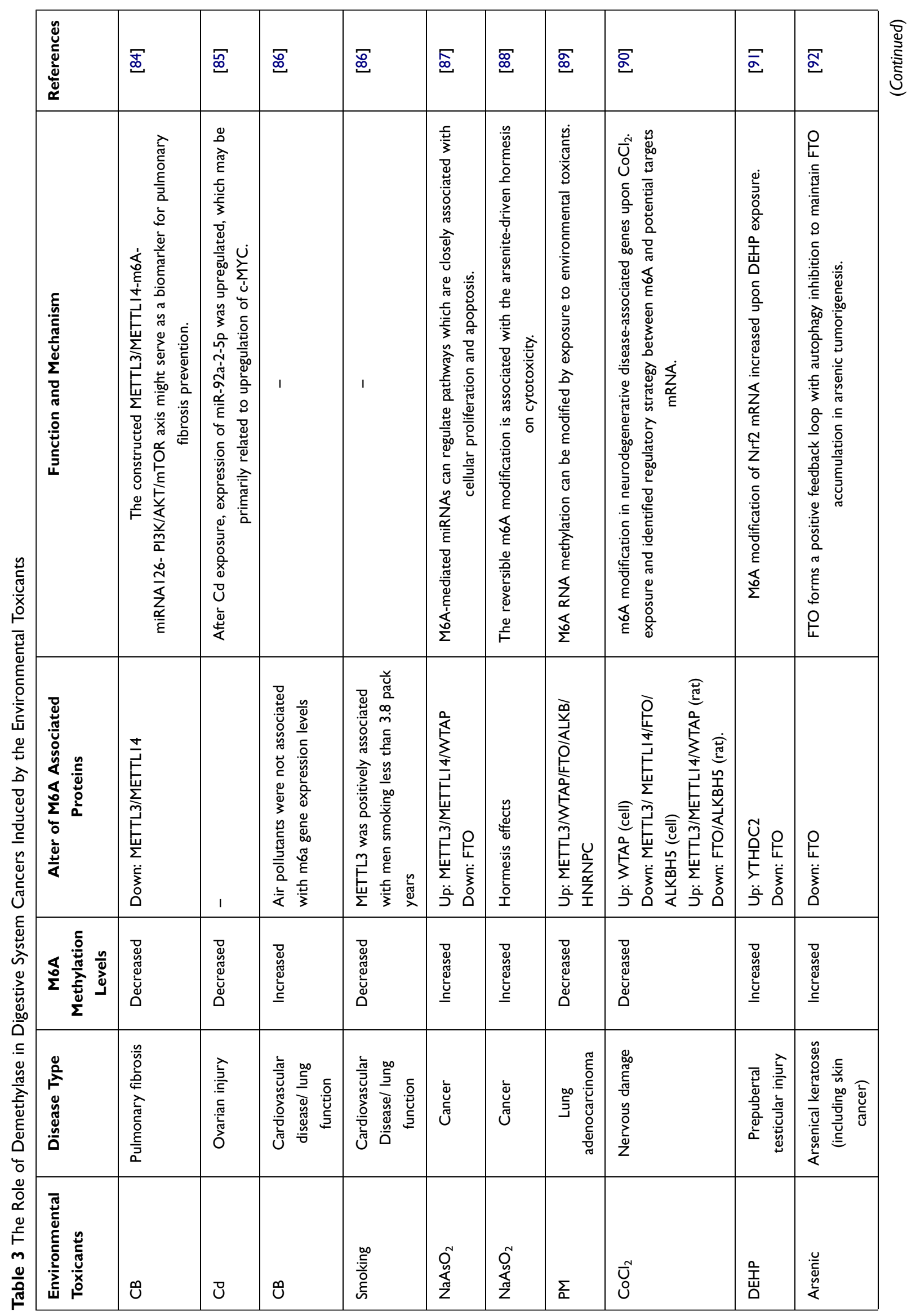




\begin{tabular}{|c|c|c|c|c|c|}
\hline 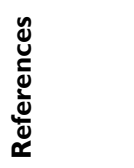 & $\bar{\Xi}$ & 写 & \multicolumn{2}{|l|}{ 沉 } & 守 \\
\hline 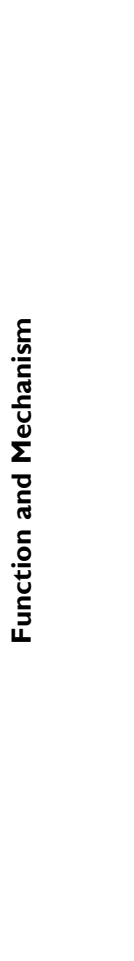 & 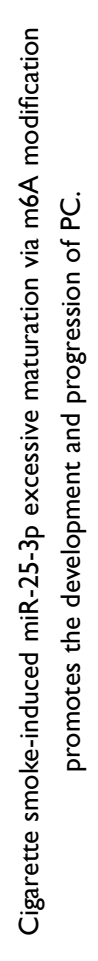 & 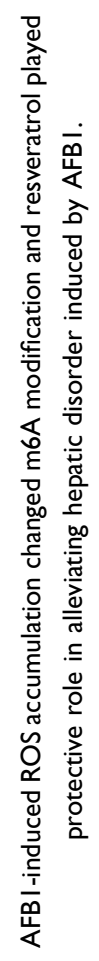 & \multicolumn{2}{|l|}{ 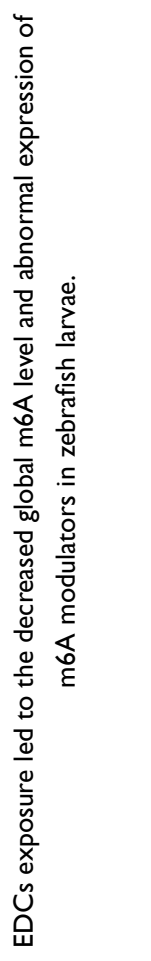 } & 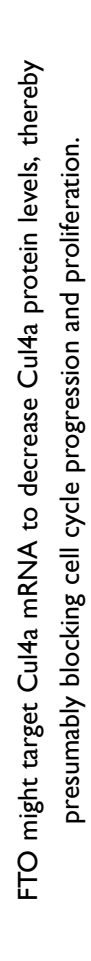 \\
\hline 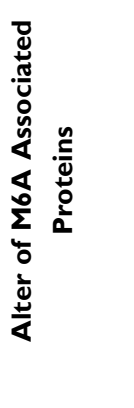 & $\begin{array}{l}\stackrel{m}{E} \\
\stackrel{E}{\omega} \\
\ddot{D}\end{array}$ & 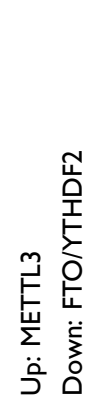 & 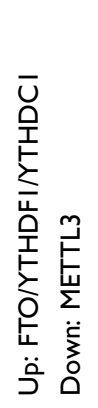 & 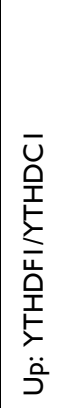 & 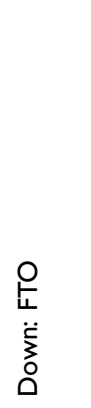 \\
\hline 竞 & 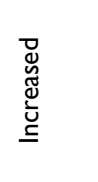 & 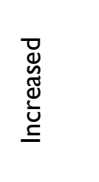 & \multicolumn{2}{|l|}{ 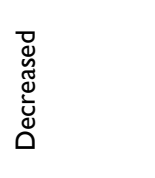 } & ' \\
\hline 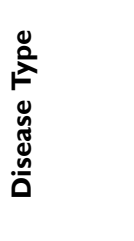 & 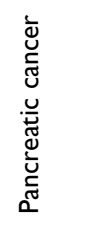 & 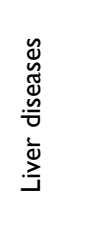 & \multicolumn{2}{|l|}{ 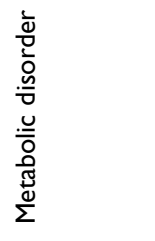 } & 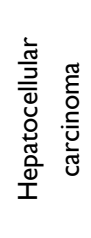 \\
\hline 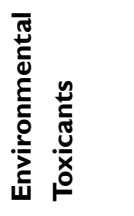 & $\mathscr{U}$ & 要 & 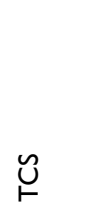 & å & 壱 \\
\hline
\end{tabular}

Herein, we summarized the demethylase roles and mechanism of FTO, ALKBH5 and ALKBH3 in digestive system cancers. We highlighted the m6A demethylase function in cancers, including the value of diagnosis, prognosis and treatment, regulating target genes to involve in tumor occurrence and development, and the association with environmental toxicants. The results of m6A demethylases are inconsistent across different studies, because the sample sources, points and approaches of studies are different. FTO and ALKBH5 are m6A demethylases, but in most studies, they represent different expression and have carcinogenic or anticarcinogenic effects on cancers, which remains to be further investigated. Though the study on the association between m6A demethylase and environmental toxicants is on the primary stage, it provides new insight into the assessment of environmental exposure, the signature of early damage, and tumor development induced by environmental toxicants, especially for prevention and diagnosis of digestive system cancers. Besides, m6A demethylases can be helpful to promote effective therapeutic strategies and develop new anticarcinogenic medicines for cancer treatment. Despite the multiple effects and potential mechanisms of m6A studies have made great progress and gained momentum in recent years. However, there are still some problems to be considered for further research. First, the different roles and mechanisms of $\mathrm{m} 6 \mathrm{~A}$ and $\mathrm{m} 6 \mathrm{~A}$ regulators in some cancers will be necessary to be proved by more center and larger samples of research. Second, the specificity and sensitivity of m6A level and its regulators as potential biomarkers for diagnosis, prognosis and environmental toxicants exposure for some cancers need to be revealed. Third, are there any other $\mathrm{m} 6 \mathrm{~A}$ related proteins that regulate the level of m6A? Fourth, studies need to offer simple handling, low cost, quick and noninvasive detection techniques of m6A for clinical application in the future.

In conclusion, m6A demethylases, FTO, ALKBH5 and ALKBH3 perform their biological function to involve in tumor occurrence and development in different ways. M6A demethylases may be potential biomarkers for diagnosis, prognosis, cancer treatment and environmental toxicant prevention in the future clinical application.

\section{Abbreviations}

M6A, N6-methyladenosine; FTO, Fat mass and obesityassociated protein; ALKBH5, AlkB homolog 5; ALKBH3, AlkB homolog 3; N, Nitrogen; A, Adenine; 3' UTR, 3' Untranslated region; MTC, Multicomponent methyltransferase complex; METTL3, Methyltransferase-like 3; METTL14, 
Methyltransferase-like 14; WTAP, Wilms tumor suppressor1-associated protein; RBM15, RNA Binding Motif Protein 15; ZC3H13, Zinc finger CCCH domain-containing protein 13; METTL16, Methyltransferase-like 16; METTL5, Methyltransferase-like 5; YTHDFs, YTH N6Methyladenosine RNA Binding Proteins; IGF2BPs, Insulinlike growth factor 2 mRNA-binding proteins; HNRNPs, Heterogeneous nuclear ribonucleoprotein; HP, Helicobacter pylori; ALKBH9, alkB homolog 9; GWAS, Genome-Wide Association Studies; SNP, Single nucleotide polymorphism; AML, Acute Myeloid Leukemia; GC, Gastric cancer; TCGA, The Cancer Genome Atlas; GEO, Gene Expression Omnibus; IHC, Immunohistochemistry; HCC, Hepatocellular carcinoma; ICC, Intrahepatic cholangiocarcinoma; CRC, Colorectal cancer; PC, Pancreatic cancer; EC, Esophageal cancer; hm6A, N6-hydroxymethyladenosine; f6A, N6formyladenosine; CB, Carbon black; Cd, Cadmium; PM, Particulate matter; DEHP, Di-(2-Ethylhexyl) phthalate; CSC, Cigarette smoke condensate; AFB1, Aflatoxin B1; ROS, Reactive oxygen species; TCS, Triclosan; BPA, Bisphenol A exposure; DEN, Diethylnitrosam.

\section{Acknowledgment}

The present study was supported by the National Natural Science Foundation of China (81972998, 81803201, 81673132).

\section{Disclosure}

The authors declare no financial competing interests or non-financial competing interests in this work.

\section{References}

1. Mattiuzzi C, Lippi G. Current cancer epidemiology. J Epidemiol Glob Health. 2019;9(4):217-222. doi:10.2991/jegh.k.191008.001

2. Wu C, Li M, Meng H, et al. Analysis of status and countermeasures of cancer incidence and mortality in China. Sci China Life Sci. 2019;62 (5):640-647. doi:10.1007/s11427-018-9461-5

3. Sung H, Ferlay J, Siegel RL, et al. Global cancer statistics 2020: GLOBOCAN estimates of incidence and mortality worldwide for 36 cancers in 185 countries. CA Cancer J Clin. 2021;71(3):209-249. doi:10.3322/caac. 21660

4. Feng RM, Zong YN, Cao SM, Xu RH. Current cancer situation in China: good or bad news from the 2018 global cancer statistics? Cancer Commun. 2019;39(1):22. doi:10.1186/s40880-019-0368-6

5. Calati R, Fang F, Mostofsky E, et al. Cancer and suicidal ideation and behaviours: protocol for a systematic review and meta-analysis. $B M J$ Open. 2018;8(8):e020463. doi:10.1136/bmjopen-2017-020463

6. Woo HH, Chambers SK. Human ALKBH3-induced m(1)A demethylation increases the CSF-1 mRNA stability in breast and ovarian cancer cells. Biochim Biophys Acta Gene Regul Mech. 2019;1862 (1):35-46. doi:10.1016/j.bbagrm.2018.10.008
7. Boccaletto P, Machnicka MA, Purta E, et al. MODOMICS: a database of RNA modification pathways. 2017 update. Nucleic Acids Res. 2018;46(D1):D303-D307. doi:10.1093/nar/gkx1030

8. Liu L, Wang Y, Wu J, Liu J, Qin Z, Fan H. N(6)-methyladenosine: a potential breakthrough for human cancer. Mol Ther Nucleic Acids. 2020;19:804-813. doi:10.1016/j.omtn.2019.12.013

9. Li Y, Wu K, Quan W, et al. The dynamics of FTO binding and demethylation from the m(6)A motifs. RNA Biol. 2019;16 (9):1179-1189. doi:10.1080/15476286.2019.1621120

10. Yang YY, Yu K, Li L, Huang M, Wang Y. Proteome-wide interrogation of small GTPases regulated by $\mathrm{N}(6)$-methyladenosine modulators. Anal Chem. 2020;92(14):10145-10152. doi:10.1021/ acs.analchem.0c02203

11. Leonetti AM, Chu MY, Ramnaraign FO, Holm S, Walters BJ. An emerging role of m6A in memory: a case for translational priming. Int J Mol Sci. 2020;21(20):7447. doi:10.3390/ijms21207447

12. Wang TY, Kong S, Tao M, Ju SQ. The potential role of RNA N6-methyladenosine in cancer progression. Mol Cancer. 2020;19 (1):88. doi:10.1186/s12943-020-01204-7

13. Fu Y, Jia G, Pang X, et al. FTO-mediated formation of N6-hydroxymethyladenosine and N6-formyladenosine in mammalian RNA. Nat Commun. 2013;4(1):1798. doi:10.1038/ncomms 2822

14. Guan KL, Liu X, Li JH, et al. Expression status and prognostic value of M6A-associated genes in gastric cancer. J Cancer. 2020;11 (10):3027-3040. doi:10.7150/jca.40866

15. Niu Y, Lin Z, Wan A, et al. RNA N6-methyladenosine demethylase FTO promotes breast tumor progression through inhibiting BNIP3. Mol Cancer. 2019;18(1):46. doi:10.1186/s12943-019-1004-4

16. Li T, Hu PS, Zuo Z, et al. METTL3 facilitates tumor progression via an $\mathrm{m}(6)$ A-IGF2BP2-dependent mechanism in colorectal carcinoma. Mol Cancer. 2019;18(1):112. doi:10.1186/s12943-0191038-7

17. Zhao W, Qi XQ, Liu LN, Ma SQ, Liu JW, Wu J. Epigenetic regulation of m(6)A modifications in human cancer. Mol Ther Nucleic Acids. 2020;19:405-412. doi:10.1016/j.omtn.2019.11.022

18. Li N, Zhan XQ. Identification of pathology-specific regulators of $\mathrm{m}$ (6)A RNA modification to optimize lung cancer management in the context of predictive, preventive, and personalized medicine. EPMA J. 2020;11(3):485-504. doi:10.1007/s13167-020-00220-3

19. Pu XW, Gu ZW, Gu ZR. ALKBH5 regulates IGF1R expression to promote the proliferation and tumorigenicity of endometrial cancer. J Cancer. 2020;11(19):5612-5622. doi:10.7150/jca.46097

20. Lobo J, Barros-Silva D, Henrique R, Jerónimo C. The emerging role of epitranscriptomics in cancer: focus on urological tumors. Genes. 2018;9(11):552. doi:10.3390/genes9110552

21. Wang J, Wang $\mathrm{J}, \mathrm{Gu} \mathrm{Q}$, et al. The biological function of $\mathrm{m} 6 \mathrm{~A}$ demethylase ALKBH5 and its role in human disease. Cancer Cell Int. 2020;20(1):347. doi:10.1186/s12935-020-01450-1

22. Machlowska J, Baj J, Sitarz M, Maciejewski R, Sitarz R. Gastric cancer: epidemiology, risk factors, classification, genomic characteristics and treatment strategies. Int J Mol Sci. 2020;21(11):4012. doi:10.3390/ijms21114012

23. Yang JD, Hainaut P, Gores GJ, Amadou A, Plymoth A, Roberts LR. A global view of hepatocellular carcinoma: trends, risk, prevention and management. Nat Rev Gastroenterol Hepatol. 2019;16 (10):589-604. doi:10.1038/s41575-019-0186-y

24. Li D, Zhu X, Li Y, Zeng X. Novel insights into the roles of RNA N (6)-methyladenosine modification in regulating gene expression during environmental exposures. Chemosphere. 2020;261:127757. doi:10.1016/j.chemosphere.2020.127757

25. Fawcett KA, Barroso I. The genetics of obesity: FTO leads the way. Trends Genet. 2010;26(6):266-274. doi:10.1016/j.tig.2010.0 2.006

26. Qin Y, Li L, Luo E, et al. Role of m6A RNA methylation in cardiovascular disease (review). Int J Mol Med. 2020;46 (6):1958-1972. doi:10.3892/ijmm.2020.4746 
27. Church C, Moir L, McMurray F, et al. Overexpression of Fto leads to increased food intake and results in obesity. Nat Genet. 2010;42 (12):1086-1092. doi:10.1038/ng.713

28. Huang X, Zhao J, Yang M, Li M, Zheng J. Association between FTO gene polymorphism (rs9939609 T/A) and cancer risk: a meta-analysis. Eur $J$ Cancer Care. 2017;26(5):e12464. doi:10.1111/ecc.12464

29. Li G, Chen Q, Wang L, Ke D, Yuan Z. Association between FTO gene polymorphism and cancer risk: evidence from 16,277 cases and 31,153 controls. Tumour Biol. 2012;33(4):1237-1243. doi:10.1007/ s13277-012-0372-9

30. Zhao J, Huang X, Yang M, Li M, Zheng J. Association between the FTOrs 8050136 polymorphism and cancer risk: a meta-analysis. Fam Cancer. 2016;15(1):145-153. doi:10.1007/s10689-015-9843-7

31. Jia G, Fu Y, Zhao X, et al. N6-methyladenosine in nuclear RNA is a major substrate of the obesity-associated FTO. Nat Chem Biol. 2011;7(12):885-887. doi:10.1038/nchembio.687

32. Wei J, Liu F, Lu Z, et al. Differential m(6)A, m(6)Am, and m(1)A demethylation mediated by FTO in the cell nucleus and cytoplasm. Mol Cell. 2018;71(6):973-985e5. doi:10.1016/j.molcel.2018.08.011

33. Li Z, Weng H, Su R, et al. FTO plays an oncogenic role in acute myeloid leukemia as a N(6)-methyladenosine RNA demethylase. Cancer Cell. 2017;31(1):127-141. doi:10.1016/j.ccell.2016.11.017

34. Xu X, Zhou E, Zheng J, et al. Prognostic and predictive value of m6a "eraser" related gene signature in gastric cancer. Front Oncol. 2021;11:631803. doi:10.3389/fonc.2021.631803

35. Silva I, Alipio C, Pinto R, Mateus V. Potential anti-inflammatory effect of erythropoietin in non-clinical studies in vivo: a systematic review. Biomed Pharmacother. 2021;139:111558. doi:10.1016/j. biopha.2021.111558

36. Zhang J, Piao HY, Wang Y, et al. To develop and validate the combination of RNA methylation regulators for the prognosis of patients with gastric cancer. Onco Targets Ther. 2020;13:10785-10795. doi:10.2147/OTT.S276239

37. Xu D, Shao W, Jiang Y, Wang X, Liu Y, Liu X. FTO expression is associated with the occurrence of gastric cancer and prognosis. Oncol Rep. 2017;38(4):2285-2292. doi:10.3892/or.2017.5904

38. Jing JJ, Zhao X, Li H, Sun LP, Yuan Y. Expression profiles and prognostic roles of m6A writers, erasers and readers in gastric cancer. Future Oncol. 2021;17(20):2605-2620. doi:10.2217/fon-2020-0630

39. Su Y, Huang J, Hu J. m(6)A RNA methylation regulators contribute to malignant progression and have clinical prognostic impact in gastric cancer. Front Oncol. 2019;9:1038. doi:10.3389/ fonc. 2019.01038

40. Yang Z, Jiang X, Zhang Z, et al. HDAC3-dependent transcriptional repression of FOXA2 regulates $\mathrm{FTO} / \mathrm{m} 6 \mathrm{~A} / \mathrm{MYC}$ signaling to contribute to the development of gastric cancer. Cancer Gene Ther. 2021;28(1-2):141-155. doi:10.1038/s41417-020-0193-8

41. He B, Li X, Hu T, Lian W, Zhang M. Construction of a lentiviral vector containing shRNA targeting ADAM17 and its role in attenuating endotoxemia in mice. Mol Med Rep. 2017;16(5):6013-6019. doi: $10.3892 / \mathrm{mmr} .2017 .7307$

42. Li Y, Zheng D, Wang F, Xu Y, Yu H, Zhang H. Expression of demethylase genes, FTO and ALKBH1, is associated with prognosis of gastric cancer. Dig Dis Sci. 2019;64(6):1503-1513. doi:10.1007/ s10620-018-5452-2

43. Jing-Nan PI, Zhang J, Wang XP, Xiong JC, Yu J. Down -regulation of FTO in human gastric cancer and its effect on cell line MGC-803 function. Basic Clin Med. 2017;37:098-911.

44. Ge L, Zhang N, Chen Z, et al. Level of N6-methyladenosine in peripheral blood RNA: a novel predictive biomarker for gastric cancer. Clin Chem. 2020;66(2):342-351. doi:10.1093/clinchem/hvz004

45. Rong ZX, Li Z, He JJ, et al. Downregulation of fat mass and obesity associated (FTO) promotes the progression of intrahepatic cholangiocarcinoma. Front Oncol. 2019;9:369. doi:10.3389/ fonc. 2019.00369
46. Liu XM, Liu JY, Xiao W, et al. SIRT1 regulates $\mathrm{N}$-6-methyladenosine RNA modification in hepatocarcinogenesis by inducing RANBP2-dependent FTO SUMOylation. Hepatology. 2020;72(6):2029-2050. doi:10.1002/hep.31222

47. Li J, Zhu LJ, Shi YH, Liu JN, Lin L, Chen X. m6A demethylase FTO promotes hepatocellular carcinoma tumorigenesis via mediating PKM2 demethylation. Am J Transl Res. 2019;11(9):6084.

48. Ye ZQ, Wang SB, Chen WY, et al. Fat mass and obesity-associated protein promotes the tumorigenesis and development of liver cancer. Oncol Lett. 2020;20(2):1409-1417. doi:10.3892/ol.2020.11673

49. Mittenbuhler MJ, Saedler K, Nolte H, et al. Hepatic FTO is dispensable for the regulation of metabolism but counteracts HCC development in vivo. Mol Metab. 2020;42:101085. doi:10.1016/j. molmet.2020.101085

50. Tsuruta N, Tsuchihashi $\mathrm{K}$, Ohmura $\mathrm{H}$, et al. RNA N6-methyladenosine demethylase FTO regulates PD-L1 expression in colon cancer cells. Biochem Biophys Res Commun. 2020;530 (1):235-239. doi:10.1016/j.bbrc.2020.06.153

51. Yue CF, Chen JR, Li ZY, Li LS, Chen JG, Guo YM. microRNA-96 promotes occurrence and progression of colorectal cancer via regulation of the AMPK alpha 2-FTO-m6A/MYC axis. J Exp Clin Cancer Res. 2020;39(1):240. doi:10.1186/s13046-020-01731-7

52. Zhang Q, Cai Y, Kurbatov V, et al. Gene alterations of N6-methyladenosine $(\mathrm{m}(6) \mathrm{A})$ regulators in colorectal cancer: a TCGA Database Study. Biomed Res Int. 2020;2020:8826456. doi: $10.1155 / 2020 / 8826456$

53. Liu X, Liu L, Dong Z, et al. Expression patterns and prognostic value of m(6) A-related genes in colorectal cancer. Am J Transl Res. 2019;11(7):3972-3991.

54. Relier S, Ripoll J, Guillorit H, et al. FTO-mediated cytoplasmic m(6)Am demethylation adjusts stem-like properties in colorectal cancer cell. Nat Commun. 2021;12(1):1716. doi:10.1038/s41467-021-21758-4

55. Tang X, Liu S, Chen D, Zhao Z, Zhou J. The role of the fat mass and obesity-associated protein in the proliferation of pancreatic cancer cells. Oncol Lett. 2019;17(2):2473-2478. doi:10.3892/ol.2018.9873

56. Zhao H, Xu Y, Xie Y, et al. m6A regulators is differently expressed and correlated with immune response of esophageal cancer. Front Cell Dev Biol. 2021;9:650023. doi:10.3389/fcell.2021.650023

57. Liu SX, Huang M, Chen ZQ, et al. FTO promotes cell proliferation and migration in esophageal squamous cell carcinoma through up-regulation of MMP13. Exp Cell Res. 2020;389(1):111894. doi:10.1016/j.yexcr.2020.111894

58. Zheng G, Dahl John A, Niu Y, et al. ALKBH5 is a mammalian RNA demethylase that impacts RNA metabolism and mouse fertility. Mol Cell. 2013;49(1):18-29. doi:10.1016/j.molcel.2012.10.015

59. Mo P, Xie S, Cai W, et al. N(6)-methyladenosine (m(6)A) RNA methylation signature as a predictor of stomach adenocarcinoma outcomes and its association with immune checkpoint molecules. J Int Med Res. 2020;48(9):300060520951405. doi:10.1177/0300060520951405

60. Zhang J, Guo S, Piao HY, et al. ALKBH5 promotes invasion and metastasis of gastric cancer by decreasing methylation of the lncRNA NEAT1. J Physiol Biochem. 2019;75(3):379-389. doi:10.1007/ s13105-019-00690-8

61. Ji L, Chen S, Gu L, Zhang X. Exploration of potential roles of m6A regulators in colorectal cancer prognosis. Front Oncol. 2020;10:768. doi: $10.3389 /$ fonc 2020.00768

62. Xu D, Shao J, Song H, Wang J. The YTH domain family of n6methyladenosine "readers" in the diagnosis and prognosis of colonic adenocarcinoma. Biomed Res Int. 2020;2020:9502560. doi:10.1155/ 2020/9502560

63. Liu T, Li CY, Jin LP, Li C, Wang L. The prognostic value of m6A RNA methylation regulators in colon adenocarcinoma. Med Sci Monit. 2019;25:9435-9445. doi:10.12659/msm.920381

64. Yang P, Wang Q, Liu A, Zhu J, Feng J. ALKBH5 holds prognostic values and inhibits the metastasis of colon cancer. Pathol Oncol Res. 2020;26(3):1615-1623. doi:10.1007/s12253-019-00737-7 
65. Guo T, Liu DF, Peng SH, Xu AM. ALKBH5 promotes colon cancer progression by decreasing methylation of the lncRNA NEAT1. Am $J$ Transl Res. 2020;12(8):4542-4549.

66. Li SS, Zhou CY, Liao R, et al. ABO blood type, smoking status, other risk factors and prognosis of pancreatic ductal adenocarcinoma. Medicine. 2020;99(14):e19413. doi:10.1097/MD.0000000000019413

67. Geng Y, Guan R, Hong W, et al. Identification of m6A-related genes and m6A RNA methylation regulators in pancreatic cancer and their association with survival. Ann Transl Med. 2020;8(6):387. doi:10.21037/atm.2020.03.98

68. Cho SH, Ha M, Cho YH, et al. ALKBH5 gene is a novel biomarker that predicts the prognosis of pancreatic cancer: a retrospective multicohort study. Ann Hepatobiliary Pancreat Surg. 2018;22(4):305-309. doi:10.14701/ahbps.2018.22.4.305

69. Tang B, Yang Y, Kang M, et al. m(6)A demethylase ALKBH5 inhibits pancreatic cancer tumorigenesis by decreasing WIF-1 RNA methylation and mediating Wnt signaling. Mol Cancer. 2020;19(1):3. doi:10.1186/s12943-019-1128-6

70. Guo X, Li K, Jiang W, et al. RNA demethylase ALKBH5 prevents pancreatic cancer progression by posttranscriptional activation of PER1 in an m6A-YTHDF2-dependent manner. Mol Cancer. 2020;19(1):91. doi:10.1186/s12943-020-01158-w

71. He Y, Hu H, Wang Y, et al. ALKBH5 inhibits pancreatic cancer motility by decreasing long non-coding RNA KCNK15-AS1 methylation. Cell Physiol Biochem. 2018;48(2):838-846. doi:10.1159/000491915

72. Tang R, Zhang Y, Liang C, et al. The role of m6A-related genes in the prognosis and immune microenvironment of pancreatic adenocarcinoma. PeerJ. 2020;8:e9602. doi:10.7717/peerj.9602

73. Xu F, Zhang Z, Yuan M, et al. M6A regulatory genes play an important role in the prognosis, progression and immune microenvironment of pancreatic adenocarcinoma. Cancer Invest. 2021;39 (1):39-54. doi:10.1080/07357907.2020.1834576

74. Wang P, Wang XT, Zheng L, Zhuang CB. Gene signatures and prognostic values of $\mathrm{m} 6 \mathrm{~A}$ regulators in hepatocellular carcinoma. Front Genet. 2020;11:540186. doi:10.3389/fgene.2020.540186

75. Chen YH, Zhao YC, Chen JR, et al. ALKBH5 suppresses malignancy of hepatocellular carcinoma via $\mathrm{m}(6)$ A-guided epigenetic inhibition of LYPD1. Mol Cancer. 2020;19(1):123. doi:10.1186/s12943-02001239-w

76. Xu LC, Pan JX, Pan HD. Construction and validation of an m6A RNA methylation regulators-based prognostic signature for esophageal cancer. Cancer Manag Res. 2020;12:5385-5394. doi:10.2147/ cmar.S254870

77. Xue J, Xiao P, Yu X, Zhang X. A positive feedback loop between AlkB homolog 5 and miR-193a-3p promotes growth and metastasis in esophageal squamous cell carcinoma. Hum Cell. 2021;34 (2):502-514. doi:10.1007/s13577-020-00458-z

78. Nagaki Y, Motoyama S, Yamaguchi T, et al. m(6) A demethylase ALKBH5 promotes proliferation of esophageal squamous cell carcinoma associated with poor prognosis. Genes Cells. 2020;25 (8):547-561. doi:10.1111/gtc. 12792

79. Roundtree IA, Evans ME, Pan T, He C. Dynamic RNA modifications in gene expression regulation. Cell. 2017;169(7):1187-1200. doi:10.1016/j.cell.2017.05.045

80. Chen Z, Qi M, Shen B, et al. Transfer RNA demethylase ALKBH3 promotes cancer progression via induction of tRNA-derived small RNAs. Nucleic Acids Res. 2019;47(5):2533-2545. doi:10.1093/nar/ gky1250

81. Ueda Y, Ooshio I, Fusamae Y, et al. AlkB homolog 3-mediated tRNA demethylation promotes protein synthesis in cancer cells. Sci Rep. 2017;7:42271. doi:10.1038/srep42271

82. Xie SH, Lagergren J. Risk factors for oesophageal cancer. Best Pract Res Clin Gastroenterol. 2018;36-37:3-8. doi:10.1016/j.bpg.2018.11.008
83. Korc M, Jeon CY, Edderkaoui M, et al. Tobacco and alcohol as risk factors for pancreatic cancer. Best Pract Res Clin Gastroenterol. 2017;31(5):529-536. doi:10.1016/j.bpg.2017.09.001

84. Han B, Chu C, Su X, et al. N(6)-methyladenosine-dependent primary microRNA-126 processing activated PI3K-AKT-mTOR pathway drove the development of pulmonary fibrosis induced by nanoscale carbon black particles in rats. Nanotoxicology. 2020;14(1):1-20. doi:10.1080/17435390.2019.1661041

85. Sun Y, Zong C, Liu J, et al. C-myc promotes miR-92a-2-5p transcription in rat ovarian granulosa cells after cadmium exposure. Toxicol Appl Pharmacol. 2021;421:115536. doi:10.1016/j.taap.2021.115536

86. Kupsco A, Gonzalez G, Baker BH, et al. Associations of smoking and air pollution with peripheral blood RNA N(6)-methyladenosine in the Beijing truck driver air pollution study. Environ Int. 2020;144:106021. doi:10.1016/j.envint.2020.106021

87. Gu S, Sun D, Dai H, Zhang Z. N(6)-methyladenosine mediates the cellular proliferation and apoptosis via microRNAs in arsenite-transformed cells. Toxicol Lett. 2018;292:1-11. doi:10.1016/j.toxlet.2018.04.018

88. Chen H, Zhao T, Sun D, Wu M, Zhang Z. Changes of RNA N (6)-methyladenosine in the hormesis effect induced by arsenite on human keratinocyte cells. Toxicol in Vitro. 2019;56:84-92. doi:10.1016/j.tiv.2019.01.010

89. Cayir A, Barrow TM, Guo L, Byun HM. Exposure to environmental toxicants reduces global N6-methyladenosine RNA methylation and alters expression of RNA methylation modulator genes. Environ Res. 2019;175:228-234. doi:10.1016/j.envres.2019.05.011

90. Tang J, Zheng C, Zheng F, et al. Global N6-methyladenosine profiling of cobalt-exposed cortex and human neuroblastoma $\mathrm{H} 4$ cells presents epitranscriptomics alterations in neurodegenerative disease-associated genes. Environ Pollut. 2020;266(Pt2):115326. doi:10.1016/j.envpol.2020.115326

91. Zhao TX, Wang JK, Shen LJ, et al. Increased m6A RNA modification is related to the inhibition of the Nrf2-mediated antioxidant response in di-(2-ethylhexyl) phthalate-induced prepubertal testicular injury. Environ Pollut. 2020;259:113911. doi:10.1016/j.envpol.2020.113911

92. Cui YH, Yang S, WEi J, et al. Autophagy of the m6A mRNA demethylase FTO is impaired by low-level arsenic exposure to promote tumorigenesis. Nat Commun. 2021;12:2183. doi:10.1038/ s41467-021-22469-6

93. Zhang J, Bai R, Li M, et al. Excessive miR-25-3p maturation via $\mathrm{N}$ (6)-methyladenosine stimulated by cigarette smoke promotes pancreatic cancer progression. Nat Commun. 2019;10(1):1858. doi:10.1038/s41467-019-09712-x

94. Wu J, Gan Z, Zhuo R, Zhang L, Wang T, Zhong X. Resveratrol attenuates aflatoxin B1-induced ROS formation and increase of $\mathrm{m}(6)$ A RNA methylation. Animals. 2020;10(4):677. doi:10.3390/ ani10040677

95. Sun L, Ling Y, Jiang J, et al. Differential mechanisms regarding triclosan vs. bisphenol A and fluorene-9-bisphenol induced zebrafish lipid-metabolism disorders by RNA-Seq. Chemosphere. 2020;251:126318. doi:10.1016/j.chemosphere.2020.126318

96. Wu S, Zhang L, Deng J, et al. A novel micropeptide encoded by Y-linked LINC00278 links cigarette smoking and AR signaling in male esophageal squamous cell carcinoma. Cancer Res. 2020;80 (13):2790-2803. doi:10.1158/0008-5472.CAN-19-3440

97. Huang Y, Yan J, Li Q, et al. Meclofenamic acid selectively inhibits FTO demethylation of m6A over ALKBH5. Nucleic Acids Res. 2015;43(1):373-384. doi:10.1093/nar/gku1276

98. Fukumoto T, Zhu HR, Nacarelli T, et al. N-6-methylation of adenosine of FZD10 mRNA contributes to PARP inhibitor resistance. Cancer Res. 2019;79(11):2812-2820. doi:10.1158/0008-5472.Can18-3592 


\section{Publish your work in this journal}

Cancer Management and Research is an international, peer-reviewed open access journal focusing on cancer research and the optimal use of preventative and integrated treatment interventions to achieve improved outcomes, enhanced survival and quality of life for the cancer patient.

The manuscript management system is completely online and includes a very quick and fair peer-review system, which is all easy to use. Visit http://www.dovepress.com/testimonials.php to read real quotes from published authors.

Submit your manuscript here: https://www.dovepress.com/cancer-management-and-research-journal 